\title{
Evaluation of High Altitude Interstitial Pulmonary Edema in Healthy Participants Using Rapid 4-View Lung Ultrasound Protocol During Staged Ascent to Everest Base Camp
}

\author{
Craig D. Nowadly, $\mathrm{MD}^{1}$; Kenneth M. Kelley, $\mathrm{MD}^{1}$; Desiree H. Crane, $\mathrm{DO}^{2,3}$; John S. Rose, MD ${ }^{1}$ \\ ${ }^{1}$ Department of Emergency Medicine, University of California at Davis, Sacramento, California; ${ }^{2}$ Steele Memorial Medical Center, Salmon, Idaho; \\ ${ }^{3}$ University of California, San Francisco (Fresno), Fresno, California
}

\begin{abstract}
Introduction-Prior research identified possible interstitial pulmonary fluid, concerning for early high altitude pulmonary edema (HAPE), in a large percentage of trekkers above $3000 \mathrm{~m}$ using a comprehensive 28-view pulmonary ultrasound protocol. These trekkers had no clinical symptoms of HAPE despite these ultrasound findings. The more common 4-view lung ultrasound protocol (LUP) is accurate in rapidly detecting interstitial edema during resource-rich care. The objective of this study was to evaluate whether the 4-view LUP detects interstitial fluid in trekkers ascending to Everest Base Camp.

Methods-Serial 4-view LUP was performed on 15 healthy trekkers during a 9-d ascent from Kathmandu to Everest Base Camp. Ascent protocols complied with Wilderness Medical Society guidelines for staged ascent. A 4-view LUP was performed in accordance with the published 2012 international consensus protocols on lung ultrasound. Symptom assessment and 4-view LUP were obtained at 6 waypoints along the staged ascent. A 4-view LUP was positive for interstitial edema if $\geq 3$ B-lines were detected in 2 ultrasound windows.

Results-A single participant had evidence of interstitial lung fluid at $5380 \mathrm{~m}$ as defined by the 4view LUP. There was no evidence of interstitial fluid in any participant below $5380 \mathrm{~m}$. One participant was evacuated for acute altitude sickness at $4000 \mathrm{~m}$ but showed no preceding sonographic evidence of interstitial fluid.
\end{abstract}

Conclusions-In this small study, sonographic detection of interstitial fluid, suggestive of early HAPE, was not identified by the 4-view LUP protocol.

Keywords: altitude sickness, acute mountain sickness, wilderness medicine, mountaineering, austere medicine, lung comets

\section{Introduction}

High altitude pulmonary edema (HAPE) is noncardiogenic interstitial edema associated with ascent above $2500 \mathrm{~m} .{ }^{1,2}$ HAPE is more common at higher altitudes, with an incidence of 0.6 to $6 \%$ above $4500 \mathrm{~m}$ and 2 to $15 \%$ above $5500 \mathrm{~m}^{3}$ Mild cases include cough or exertional dyspnea. Severe cases are associated with high morbidity and mortality and require costly evacuation to

Corresponding author: Craig D. Nowadly, MD, Department of Emergency Medicine, PSSB 2100, U.C. Davis Medical Center, 4150 V Street, Sacramento, CA 95817; e-mail: cnowadly@gmail.com.

Submitted for publication May 2020.

Accepted for publication March 2021. lower altitudes. ${ }^{4,5}$ Although chest radiograph is commonly used to assess for HAPE in high-resource settings, few imaging modalities that can be used to assist in the diagnosis of HAPE are available in austere situations. ${ }^{6}$ High-resolution, point-of-care ultrasound units have emerged as useful diagnostic adjuncts to assess many pulmonary conditions, including pneumothorax and interstitial fluid, in critical and emergency care. ${ }^{7,8}$ The characteristic sonographic lung findings of interstitial fluid are termed sonographic B-lines or B-pattern., B-lines are comet tail-like reverberation artifacts that represent increased interstitial fluid within the lung parenchyma. In most situations, the number of B-lines generally correlates to the degree of interstitial fluid and has been shown to be useful in determining the severity 
and clinical course of several conditions with increased interstitial fluid content, among which pulmonary edema and congestive heart failure are well described. ${ }^{10}$ More recently, ultrasound has shown clinical utility in detecting the interstitial fluid associated with HAPE. ${ }^{11,12}$

The 2012 international pulmonary ultrasound guidelines adopted 2 primary technical protocols. ${ }^{13}$ The first is a 28-view, comprehensive protocol that provides a detailed view of both lung fields and cardiac windows. This technique also employs a semiquantitative scoring system to assess changes between examinations. The second technique is a 4-view (8-zone) lung ultrasound protocol (LUP). This protocol uses 4 views of each lung in the anterolateral plane. This technique is well described in the literature and is generally considered the preferred technique for most emergency and critical care situations; it is faster and more practical because the patient can remain supine or recumbent during the entire examination.

A previous study suggested that trekkers at altitude may have detectable B-lines on comprehensive 28-view lung ultrasound in the absence of frank pulmonary symptoms or changes on pulmonary auscultation. ${ }^{14}$ This suggested that participants may have underlying interstitial pulmonary edema but did not meet classic definitions of HAPE. We evaluated whether the more common 4-view LUP examination would detect similar findings on recreational trekkers traveling to Everest Base Camp (EBC).

\section{Methods}

\section{PARTICIPANT ENROLLMENT}

This was an observational cohort study conducted during April and May 2015. Participants were selected from a professionally guided, recreational trek to EBC from Kathmandu, Nepal. The study was approved by the University of California at Davis Institutional Review Board. Participants were recruited for participation in the study during the pretrek orientation in the United States. Written informed consent was obtained.

Study exclusion criteria included history of pulmonary or cardiovascular disease, history of hypertension (defined as a systolic blood pressure $>140 \mathrm{~mm} \mathrm{Hg}$ and/or a diastolic blood pressure $>90 \mathrm{~mm} \mathrm{Hg}$ ), history of HAPE, recent trek or prolonged exposure $(>1 \mathrm{wk})$ to high altitude $(>2000 \mathrm{~m}$ ) in the $30 \mathrm{~d}$ before study involvement, and those who were native Nepalese Sherpa or porters. No participants were excluded from participation based on these criteria. All received physician clearance for participation in high altitude activity independent of participation in this study.

\section{ASCENT}

Wilderness Medical Society guidelines for staged ascent were followed during the trek. ${ }^{15}$ Above $3000 \mathrm{~m}$, daily elevations in sleeping altitude were not increased by greater than approximately $500 \mathrm{~m}$. A rest day was included with each increase in altitude of $1000 \mathrm{~m}$ above $3000 \mathrm{~m}$, which equaled every third or fourth day. Approximate trek altitudes and locations can be seen in Figure 1. During each day of altitude gain, participants ascended an additional 100 to $200 \mathrm{~m}$ before descent to sleeping altitudes to aid in acclimatization. All participants were prophylactically given $125 \mathrm{mg}$ acetazolamide orally twice daily throughout the study in accordance with Wilderness Medical Society recommendations. The first dose was taken $1 \mathrm{~d}$ before commencing activity.

\section{DATA COLLECTION}

Participants had study-related events measured 6 times throughout the course of the ascending phase of the trek. Baseline measurements were obtained in Kathmandu (altitude $1400 \mathrm{~m}$ ) and the subsequent 5 measurements during various portions of the ascent phase. Measurements were obtained each evening immediately after the evening meal during rest periods on predetermined stops along the ascent. Vital signs included blood pressure, heart rate, and pulse oximetry using a portable pulse oximeter (Nellcor portable pulse oximeter PM10, Medtronic Minneapolis, MN). Participants completed the modified Borg dyspnea scale (MBDS) and the Lake Louise acute mountain sickness score (LLS) before each LUP for a subjective evaluation of clinical symptoms associated with high altitude exposure. Participants could rate perceived dyspnea with an MBDS between 0 (no dyspnea, asymptomatic) and 10 (maximal work of breathing, severe dyspnea) for their current work of breathing. Participants provided an LLS between 0 (asymptomatic) and 3 (severe, incapacitating) for each of the following categories: headache, gastrointestinal symptoms, fatigue, lightheadedness, and difficulty sleeping. The LLS protocol was used because this study was performed before the 2018 update, which removed sleep disturbance as a component. Although acute mountain sickness (AMS) and HAPE are independent clinical events with differing pathophysiology, it is common to include AMS symptoms when evaluating for maladaptive acclimatization conditions.

Pulmonary ultrasound was performed using the previously described 4-window LUP. ${ }^{16}$ This protocol uses 2 anterior views, upper and lower, at the midclavicular line and 2 lateral views, upper and lower, at the midaxillary line. These are done bilaterally for a total of 8 zones of the pulmonary fields. All examinations were performed 


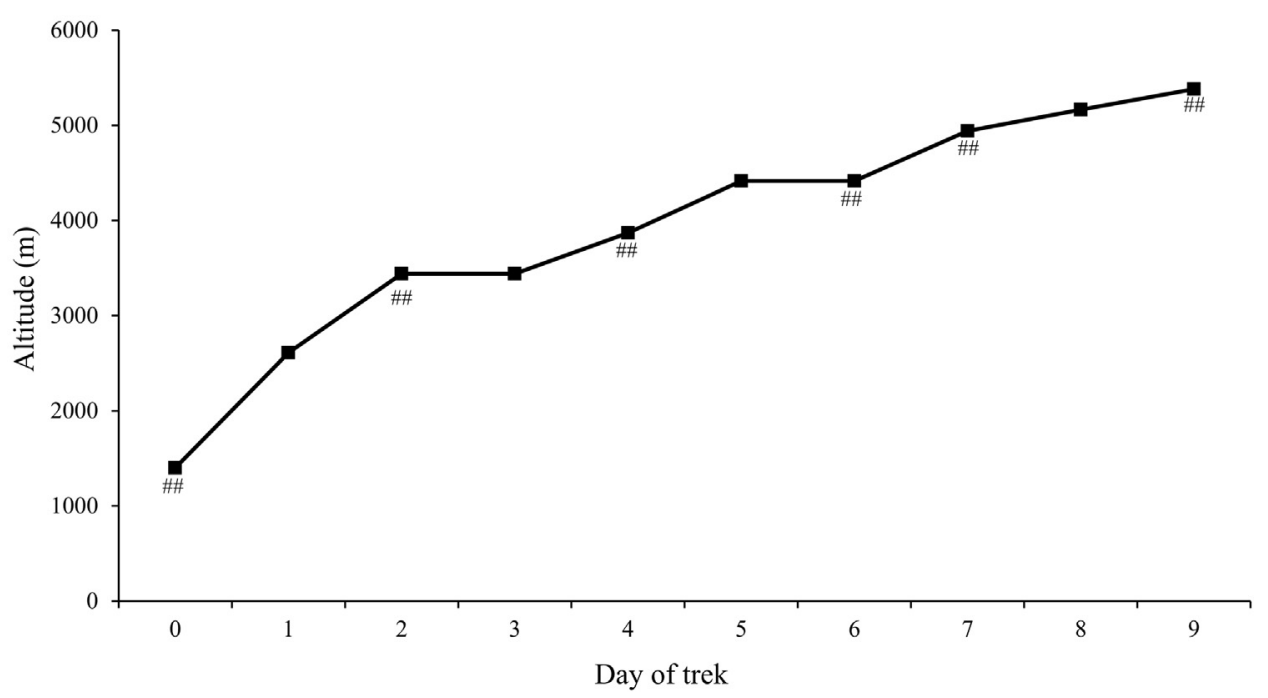

Figure 1. Approximate daily trek altitudes and locations. Day on which 4-view lung ultrasound protocol were performed are marked with \#\#. Location by day: 0 - Kathmandu, 1 - Phakding, 2 - Namche Bazaar, 3 - Namche Bazaar, 4 - Tengboche, 5 - Dingboche, 6 - Dingboche, 7 Lobuche, 8 - Gorak Shep, 9 - Everest Base Camp.

using a portable ultrasound unit (MicroMaxx Ultrasound System; FUJIFILM SonoSite, Bothell, WA). Examinations were performed using a 1 to $5 \mathrm{MHz}$ phased array ultrasound transducer at $14 \mathrm{~cm}$ depth (P17 Phased Array, FUJIFILM SonoSite). Examinations were performed with the patient in a recumbent or supine position and after no exertional activity had been performed for at least $30 \mathrm{~min}$. A single, board-certified emergency medicine physician performed the ultrasonography during this study using the same ultrasound unit, transducer, and examinatino settings. The sonographer/physician (JSR) had extensive clinical and research experience with emergency ultrasound. Images were labeled using a unique participant identifier and a corresponding lung location. Video recordings of $5 \mathrm{~s}$ in length were archived until completion of the study. The ultrasound batteries were recharged daily using portable solar panels.

\section{ANALYSIS}

On return, the archived ultrasound video recordings were independently reviewed by 2 board-certified emergency medicine physicians (KMK, DHC) with fellowship training in emergency ultrasonography and clinical experience with the 4-view LUP. The reviewers were blinded to the participant number, reported symptoms, chest location, and altitude associated with each recording. Ultrasonography B-lines were predefined as long, wide echogenic artifacts that originate from the pleural line and cross the ultrasound screen. Each recording was reviewed and the number of B-lines was documented. Reviewers classified each recording as having 0,1 to 2 , or 3 or more B-lines. A 4view LUP was considered positive if any 2 of the patient's 4 ultrasound recordings contained 3 or more B-lines in a longitudinal plane between 2 ribs, in compliance with the international definition of interstitial edema in previous ultrasound literature. ${ }^{9,13,17}$ Any discrepancies between the 2 reviewers were reassessed by both physicians and consensus was reached.

Continuous variables are presented as mean \pm SD. Discrete variables are presented as median (interquartile range). Interrater reliability was assessed using Cohen's kappa coefficient. Statistical analysis was performed using commercial software (STATA version 14.0; Stata Corp., College Station, TX).

\section{Results}

Fifteen participants were recruited: 11 male and 4 female. The median age of participants was 42 (IQR 34-52) y. Vital signs, oxygen saturation, LLS, and MBDS are presented by altitude in Table 1 . As expected, participants' subjective report of acute altitude-related symptoms increased with the change in altitude. Two-thirds (10 of 15) of participants were asymptomatic (LLS of 0) during baseline collection at $1400 \mathrm{~m}$. However, all participants were mildly symptomatic on arrival at EBC at $5380 \mathrm{~m}$ with a median LLS of 3 (IQR 2-4). All 15 respondents reported an MBDS of 0 during baseline collection. Four participants reported an MBDS of 1 at $5380 \mathrm{~m}$. Participants had a progressive decrease in 
Table 1. Participant vital signs, subjective modified Borg dyspnea scale, Lake Louise acute mountain sickness score, and participant ultrasounds

\begin{tabular}{|c|c|c|c|c|c|c|}
\hline & $\begin{array}{c}\text { Kathmandu } \\
1400 \mathrm{~m}\end{array}$ & $\begin{array}{c}\text { Namche Bazaar } \\
3440 \mathrm{~m}\end{array}$ & $\begin{array}{l}\text { Tengboche } \\
3870 \mathrm{~m}\end{array}$ & $\begin{array}{c}\text { Dingboche } \\
4415 \mathrm{~m}\end{array}$ & $\begin{array}{l}\text { Lobuche } \\
4940 \mathrm{~m}\end{array}$ & $\begin{array}{l}\text { Everest Base } \\
\text { Camp } 5380 \mathrm{~m}\end{array}$ \\
\hline Participants, $\mathrm{n}$ & 15 & 15 & 15 & 14 & 14 & 14 \\
\hline Lake Louise score & $0(0-1)$ & $1(0-1)$ & $1(1-1)$ & $1.5(1-2)$ & $2.5(2-3)$ & $3(2-4)$ \\
\hline Modified Borg score & $0(0-0)$ & $0(0-0)$ & $0(0-0)$ & $0(0-0)$ & $0(0-0)$ & $0(0-0.75)$ \\
\hline $\begin{array}{l}\text { Systolic blood pressure } \\
\text { (mm } \mathrm{Hg})\end{array}$ & $133 \pm 12$ & $134 \pm 14$ & $136 \pm 18$ & $133 \pm 12$ & $131 \pm 10$ & $130 \pm 10$ \\
\hline $\begin{array}{l}\text { Diastolic blood pressure } \\
\quad(\mathrm{mm} \mathrm{Hg})\end{array}$ & $77 \pm 22$ & $77 \pm 11$ & $74 \pm 8$ & $76 \pm 7$ & $77 \pm 8$ & $73 \pm 6$ \\
\hline Heart rate $\left(\right.$ beats $\cdot \mathrm{min}^{-1}$ ) & $76 \pm 14$ & $93 \pm 14$ & $79 \pm 12$ & $79 \pm 12$ & $83 \pm 13$ & $91 \pm 16$ \\
\hline $\begin{array}{l}\text { Respiratory rate } \\
\quad\left(\text { breaths } \cdot \mathrm{min}^{-1}\right)\end{array}$ & $18 \pm 1$ & $16 \pm 2$ & $18 \pm 4$ & $18 \pm 5$ & $21 \pm 4$ & $22 \pm 3$ \\
\hline Oxygen saturation $(\%)$ & $97 \pm 2$ & $94 \pm 2$ & $91 \pm 5$ & $86 \pm 6$ & $82 \pm 6$ & $77 \pm 6$ \\
\hline $\begin{array}{l}\text { Positive 4-view } \\
\text { ultrasound protocol }\end{array}$ & 0 & 0 & 0 & 0 & 0 & 1 \\
\hline No B-lines detected & 14 & 14 & 14 & 10 & 9 & 12 \\
\hline
\end{tabular}

Continuous variables are presented as mean \pm SD. Discrete variables are presented as median (interquartile range). A positive 4-window lung ultrasound protocol was defined by any examination with $>3$ B-lines on 2 or more video recordings. A study was considered to have no B-lines detected when 0 B-lines were identified after review of all video images.

oxygen saturation and increase in respiratory rate, consistent with altitude exposure.

The results of ultrasound examinations are shown in Table 1 . The video views obtained were satisfactory for assessment by both reviewers. A Cohen kappa coefficient of 0.72 was measured between the 2 reviewers. There was no evidence of interstitial edema in any participant below $5380 \mathrm{~m}$. At $5380 \mathrm{~m}$, a single patient met the 4-window LUP definition of positive for interstitial edema but reported no significant pulmonary symptoms. B-lines were detected in the bilateral lower pulmonary windows. This patient had no evidence of B-lines on any ultrasound below $5380 \mathrm{~m}$. At the time of the positive finding, this participant reported an MBDS of 0 but an LLS of 6, consistent with mild altitude sickness. In the majority of patients at each altitude, 0 Blines were detected during all ultrasounds.

One female trekker was medically evacuated by helicopter at approximately $4000 \mathrm{~m}$ for worsening AMS (headache, nausea, and fatigue) and inability to continue because of symptoms. This participant's ultrasound examinations at 1400, 3440, and $3870 \mathrm{~m}$ showed no evidence of B-lines. It is unclear whether this participant was answering the symptom instruments honestly given an underlying reluctance to terminate her trek. Owing to her medical evacuation, the data presented for subsequent altitudes contain the remaining 14 participants.

\section{Discussion}

We sought to evaluate whether the commonly used 4view LUP examination would detect pulmonary fluid in healthy recreational trekkers traveling to $\mathrm{EBC}$ from Kathmandu. We were unable to detect evidence of interstitial pulmonary fluid using the 4-view LUP. A single participant had a positive 4-window LUP examination consistent with interstitial fluid while at EBC at $5380 \mathrm{~m}$ but reported no pulmonary symptoms.

The 28-view, comprehensive lung ultrasound is considered the most inclusive protocol for lung ultrasound by international guidelines. ${ }^{13}$ The original protocol involves a pulmonary and cardiac examination with ultrasonographic assessment of 28 chest locations. ${ }^{18}$ The total number of B-lines identified in each field are summed to obtain a comet tail score. Using the 28-view, comprehensive lung ultrasound, previous research reported that nearly all trekkers at altitudes of $>3000 \mathrm{~m}$ develop interstitial edema on portable ultrasonography despite no reported dyspnea or change in lung auscultation, possibly suggesting early HAPE. ${ }^{14}$ It was noted that a large majority of participants (15 of 18 at 3340 m; 18 of 18 at $4790 \mathrm{~m}$ ) had a comet tail score of $>5$ detected during the pulmonary examination at altitude. ${ }^{14}$ However, the authors did not report the more commonly accepted criteria for a positive examination, which is 3 or more B-lines in 2 or more windows. ${ }^{13,16}$

We chose to evaluate the 4-view LUP given that, in emergency situations in austere environments, it would likely be the preferred technique because it is faster, the patient can be supine or recumbent, and many emergency and critical care providers are familiar with the technique. Additionally, the examination requires less battery power, which is ideal in austere environments. In clinical practice, it has been found to be accurate in the detection 
of interstitial lung fluid and pulmonary edema in both ambulatory $^{19,20}$ and hospitalized ${ }^{21-23}$ patients with congestive heart failure. Furthermore, the 4-view LUP is advocated in emergency and critical care situations. ${ }^{13,16,17}$

Although we expected fewer B-lines would be detected by the 4-view LUP compared to the 28-view comprehensive protocol given the reduced number of windows, we detected far fewer total B-lines at altitude than expected. Prior research detected the presence of Blines in $100 \%$ of trekkers at an altitude of $5130 \mathrm{~m}$ using the 28 -view protocol. ${ }^{14}$ However, at a similar altitude, our study detected B-lines in only 2 of the 14 participants, of whom only 1 case met the international consensus definition of a B-pattern consistent with interstitial fluid. By contrast, no B-lines were detected in any of the other 12 trekkers at EBC. Although we performed fewer total ultrasounds, this does not fully explain the discrepancy between the studies. It remains unknown whether the cohorts had different rates of interstitial edema, whether findings are related to the method of detection, or if findings were influenced by other factors (eg, acclimatization protocol, speed of ascent, vascular remodeling).

\section{LIMITATIONS}

There are many known and unknown confounding variables when attempting research in austere environments, and this study had several limitations. First, the sonographer was not blinded to the hypothesis while performing the ultrasounds, which could inadvertently affect ultrasound quality and B-line detection. Second, interstitial fluid associated with HAPE may have distribution characteristics that limit its detection by the 4-view LUP until more fluid is present. The maximum altitude achieved during this study was $5380 \mathrm{~m}$. We cannot draw conclusions about the development or detection of HAPE at higher altitudes. Furthermore, the risk factors for the development of HAPE remain poorly understood. It is believed that genetics play a substantial role in determining HAPE risk. ${ }^{24}$ Given our small sample size of 15 participants, it is possible that a cohort with a different genetic risk would have developed interstitial edema at a different rate.

Finally, a notable limitation to this study is the lack of prior, prospective comparisons between the 4-view LUP and 28-view comprehensive protocol. Although the 4view LUP is commonly used by providers, we know of no study that has directly compared and quantified differential B-line detection between the 2 protocols. We were unable to perform both the 4-view and 28-view protocols during our study owing to limitations on ultrasound battery life. Additional research will be required to further quantify the difference between these techniques in austere conditions and further explore the presence of possible interstitial edema in the absence of frank pulmonary symptoms.

\section{Conclusions}

In this small study, sonographic evidence of interstitial lung fluid was not detected by the 4-view LUP examination in healthy participants during a trek to an altitude of $5380 \mathrm{~m}$.

Acknowledgments: We thank Sanjay Sexton and David Breashears for their encouragement for this project. We thank Fujifilm Sonosite for providing the MicroMaxx Ultrasound System to facilitate the completion of this project.

Author Contributions: Study concept and design (JSR); acquisition of the data (JSR); analysis of the data (CDN, KMK, JSR, DHC); drafting of the manuscript (CDN); critical revision of the manuscript (CDN, KMK, JSR); and approval of final manuscript (CDN, KMK, JSR, DHC).

Financial/Material Support: None.

Disclosures: None.

\section{References}

1. Hackett PH, Roach RC. High-altitude illness. N Engl J Med. 2001;345(2):107-14.

2. Houston CS. Acute pulmonary edema of high altitude. N Engl J Med. 1960;263(10):478-80.

3. Jensen JD, Vincent AL. High Altitude Pulmonary Edema. [Updated 2020 Aug 16]. In: StatPearls [Internet]. Treasure Island (FL): StatPearls Publishing; 2021 Jan-. Available from: https://www.ncbi.nlm.nih.gov/books/NBK430819/.

4. Paralikar SJ. High altitude pulmonary edema-clinical features, pathophysiology, prevention and treatment. Indian J Occup Environ Med. 2012;16(2):59-62.

5. Gabry AL, Ledoux X, Mozziconacci M, Martin C. Highaltitude pulmonary edema at moderate altitude $(<2,400 \mathrm{~m} ; 7$, 870 feet): a series of 52 patients. Chest. 2003;123(1):49-53.

6. Nowadly CD, Solomon AJ, Burke SM, Rose JS. Evaluation of serial chest radiographs of high-altitude pulmonary edema requiring medical evacuation from South Pole Station, Antarctica: from diagnosis to recovery. Mil Med. 2020.

7. Volpicelli G. Sonographic diagnosis of pneumothorax. Intensive Care Med. 2011;37(2):224-32.

8. Volpicelli G, Melniker LA, Cardinale L, Lamorte A, Frascisco MF. Lung ultrasound in diagnosing and monitoring pulmonary interstitial fluid. Radiol Med. 2013;118(2):196-205.

9. Lichtenstein DA, Mezière GA. Relevance of lung ultrasound in the diagnosis of acute respiratory failure: the BLUE protocol. Chest. 2008;134(1):117-25.

10. Liteplo AS, Marill KA, Villen T, Miller RM, Murray AF, Croft PE, et al. Emergency thoracic ultrasound in the differentiation of the etiology of shortness of breath (ETUDES): sonographic B-lines and N-terminal pro-brain- 
type natriuretic peptide in diagnosing congestive heart failure. Acad Emerg Med. 2009;16(3):201-10.

11. Wimalasena Y, Windsor J, Edsell M. Using ultrasound lung comets in the diagnosis of high altitude pulmonary edema: fact or fiction? Wilderness Environ Med. 2013;24(2):159-64.

12. Yang W, Wang Y, Qiu Z, Huang X, Lv M, Liu B, et al. Lung ultrasound is accurate for the diagnosis of high-altitude pulmonary edema: a prospective study. Can Respir J. 2018;2018:5804942.

13. Volpicelli G, Elbarbary M, Blaivas M, Lichtenstein DA, Mathis G, Kirkpatrick AW, et al. International evidencebased recommendations for point-of-care lung ultrasound. Intensive Care Med. 2012;38(4):577-91.

14. Pratali L, Cavana M, Sicari R, Picano E. Frequent subclinical high-altitude pulmonary edema detected by chest sonography as ultrasound lung comets in recreational climbers. Crit Care Med. 2010;38(9):1818-23.

15. Luks AM, Auerbach PS, Freer L, Grissom CK, Keyes LE, McIntosh SE, et al. Wilderness Medical Society clinical practice guidelines for the prevention and treatment of acute altitude illness: 2019 update. Wilderness Environ Med. 2019;30(4S):S3-18.

16. Malbrain M, Tavernier B, Haverals S, Slama M, Vieillard-Baron A, Wong A, et al. Executive summary on the use of ultrasound in the critically ill: consensus report from the 3rd Course on Acute Care Ultrasound (CACU). Anaesthesiol Intensive Ther. 2017;49(5):393-411.

17. Volpicelli G, Mussa A, Garofalo G, Cardinale L, Casoli G, Perotto F, et al. Bedside lung ultrasound in the assessment of alveolar-interstitial syndrome. Am $J$ Emerg Med. 2006;24(6):689-96.

18. Jambrik Z, Monti S, Coppola V, Agricola E, Mottola G, Miniati M, et al. Usefulness of ultrasound lung comets as a nonradiologic sign of extravascular lung water. Am J Cardiol. 2004;93(10):1265-70.

19. Platz E, Lewis EF, Uno H, Peck J, Pivetta E, Merz A, et al. Detection and prognostic value of pulmonary congestion by lung ultrasound in ambulatory heart failure patients. Eur Heart J. 2016;37(15):1244-51.

20. Dwyer KH, Merz AA, Lewis E, Claggett B, Crousillat DR, Lau ES, et al. Pulmonary congestion by lung ultrasound in ambulatory patients with heart failure with reduced or preserved ejection fraction and hypertension. J Card Fail. 2018;24(4):219-26.

21. Platz E, Campbell RT, Claggett B, Lewis EF, Groarke JD, Docherty KF, et al. Lung ultrasound in acute heart failure: prevalence of pulmonary congestion and short- and long-term outcomes. JACC Heart Fail. 2019;7(10):849-58.

22. Cogliati C, Casazza G, Ceriani E, Torzillo D, Furlotti S, Bossi I, et al. Lung ultrasound and short-term prognosis in heart failure patients. Int J Cardiol. 2016;218:104-8.

23. Enghard P, Rademacher S, Nee J, Hasper D, Engert U, Jorres A, et al. Simplified lung ultrasound protocol shows excellent prediction of extravascular lung water in ventilated intensive care patients. Crit Care. 2015;19(1):36.

24. Mortimer H, Patel S, Peacock AJ. The genetic basis of high-altitude pulmonary oedema. Pharmacol Ther. 2004;101(2):183-92. 